\title{
Research on tram signal priority control scheme design and implementation
}

\author{
LI Dehui ${ }^{1, a}$, LUO Meiyin ${ }^{2, b}$ and KANG Shuyan ${ }^{3, c}$ \\ ${ }^{1}$ Zhuhai Campus, Beijing Institute of Technology, Zhuhai, Guangdong, China \\ ${ }^{2}$ SCUT software company, Guangzhou, Guangdong, China \\ ${ }^{3}$ S Zhuhai Campus, Beijing Institute of Technology, Zhuhai, Guangdong, China \\ anewdhl@yeah.net, ${ }^{b} 529567877 @ q q . c o m,{ }^{c} 519075300 @ q q . c o m$
}

\begin{abstract}
Keywords: Modern trams, signal priority strategy, active priority, simulation
Abstract. With the increase the traffic congestion, it is urgent to develop the bus priority control to improve the public transit attractiveness; Recently the application of the tram has increased the complexity of the intersection priority control. The priority traffic control strategy can reduce the delay of the tram at the intersection, so as to effectively ensure the punctuality of the tram. In this paper, based on the control theories, the tram signal control process is explored and studies, This research develops and tests the concept of active priority control and as a strategy for providing priority for tram vehicles, calibrated the control parameters of the analysis and tested with VisVAP module simulation platform. The comparison with fixed-time control strategy result in substantial improvements to transit travel time and regularity with negligible impacts to private traffic, and is found to be more effective than simple preemption.
\end{abstract}

\section{Introduction}

Modern trams have the characteristics of the faster speed, higher comfort, operating time more acute. In recent years, City intersection's time and space resources are short and city intersection is the vital bottleneck of urban road. How to make scientific and reasonable signal priority control of trams is important means to assure the tram fast, on time and also is the embodiment of the bus priority.

For signal priority both at domestic and abroad has made beneficial research, such as literature 1 and 2 introduced the main signal under the condition of different way the tram system[1,2]. Literature 3 introduced the progress in the field of bus of signal priority strategy, and the influence of the accuracy of travel time prediction of priority control[3]. Literature 4 analyzed the trams various characteristics of in capacity, section layout, operating system, environmental impact, investment etc. and compared the advantages with disadvantages and analyzed the adaptability of the area in our country[4]. Literature 5 researched Shenyang tram vehicle running characteristics and through the minimum green light time, green light extension timing elements are discussed[5].Literature 6 designed the control strategy in the arterial road intersection and do the test in the field, but there are still rooms for improvement in the extension of the green time or in the compression green time[6]. Literature 7 in order to reduce average intersection delay as the goal and structure to traffic, vehicle arrival and line up for the independent variable dynamic function so as to achieve the aim of priority control[7]. Literature 8, for example, in Nottingham's trams, for the design of the control logic and add the UTC controller to improve the detection accuracy [8]. Zhuhai trams line took the lead in technology abroad without borders ground power supply. Cars adopted advanced technology such as low floor steel wheel rail vehicles, without catenary ground power supply system.

This paper analyzes Zhuhai trams priority control strategy and gets the situation is suitable for Zhuhai and applies to Meihua east road - Chinese redbud road intersection of priority control scheme, then determines the implementation timing plan. The control strategy can be applied to other intersections. 


\section{Trams signal priority scheme design}

Timing control signal design. Signal timing calculation needed parameters including signal cycle(In this paper, optimum signal cycle is Webster signal cycle), signal phase, green split, amber time, full red time, green light interval, total loss of signal time, the effective green light time. Fixed-time control timing scheme are set by the eight parameters and the investigation of traffic volume. The amber time takes $3 \mathrm{~s}$, full red time takes $0 \mathrm{~s}$, the total loss of time takes $15 \mathrm{~s}$. Suitable conditions are $\mathrm{Y}<$ 0.85 , fixed-time control timing scheme is as follows:

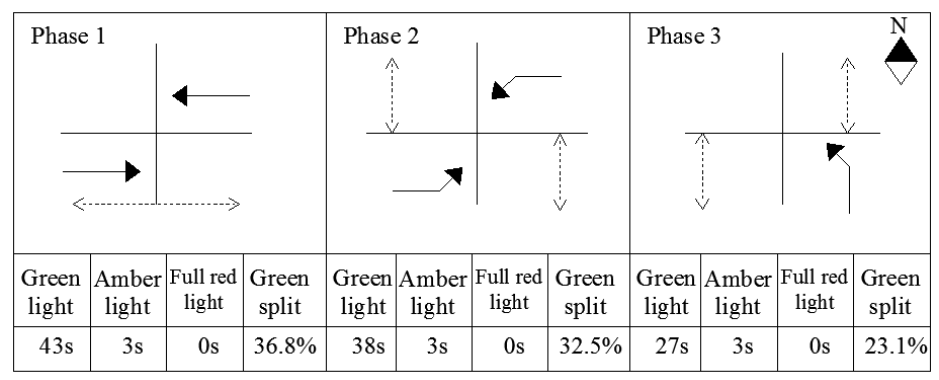

Fig.1 Fixed-time control timing scheme

Detector setup scheme. In order to realize the active priority inductive control, the road should be set sensors, the trams import lanes are laid three kinds of sensor in this scheme, the layout is shown in Fig. 2.The first kind of demand detector including detector 1 and 2.This kind of detector can scan and analyze to calculate the speed of tram. Trams driving through a second detector will give priority to signal to control signal, and through the established set for decisions. The second kind of stop line detector which is the detector 3.The type of detector set purpose is to confirm the trams have entered the signal intersection. The third kind of cancellation detector which is the detector 4.This kind of detector set purpose is to confirm that the trams have left signal intersection smoothly, then signal priority control is over.

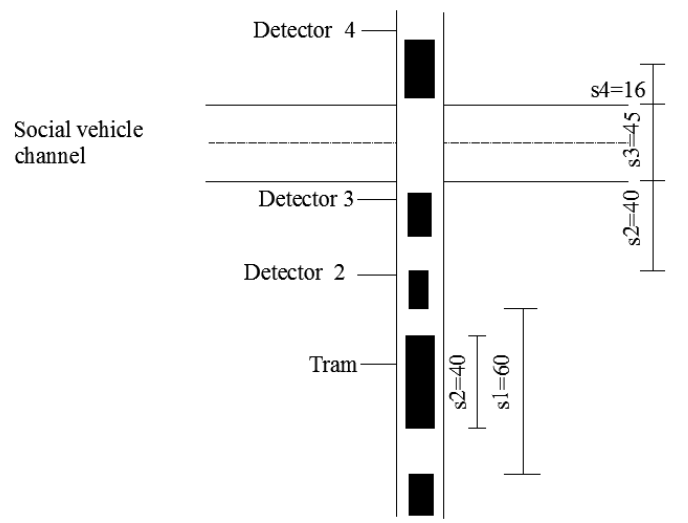

Fig.2 Schematic diagram of detector arrangement

Where L represents the length of tram; S1 represents the space between detector 1 and detector 2; S2 represents the distance between detector 2 and the intersection conflict point; S3 represents the length between tram lines and intersection flat within the scope; S4 represents the safety distance of the trams leave intersection scope, which is detector 4 from the intersection of space.

Additional real-time compensation. The most core technology in active priority control scheme is additional real-time compensation. The difference between this and the general timing is providing synchronous release of trams and covering loss of phase length which is occupied by trams. The implement of compensating the phase is the green passage of time which is occupied accounting for half of the green light of logic implementation. Such control ideas to avoid the trams start times too much and the same phase of passing time takes too long to logic errors. Modeling control phase process as shown in Fig. 3. Then, the phase 3 and phase 2 have conflict with trams phases, phase 1 is the tram phase. The key is $\mathrm{C} 1$ (whether trams pass the demand), $\mathrm{C} 2$ (whether the phase is over), $\mathrm{C} 3$ (whether the tram have left conflict areas), $\mathrm{C} 4$ (whether or not to return to this phase for second time), C5 (whether when the phase is cut off, the passage of time is more than half). 


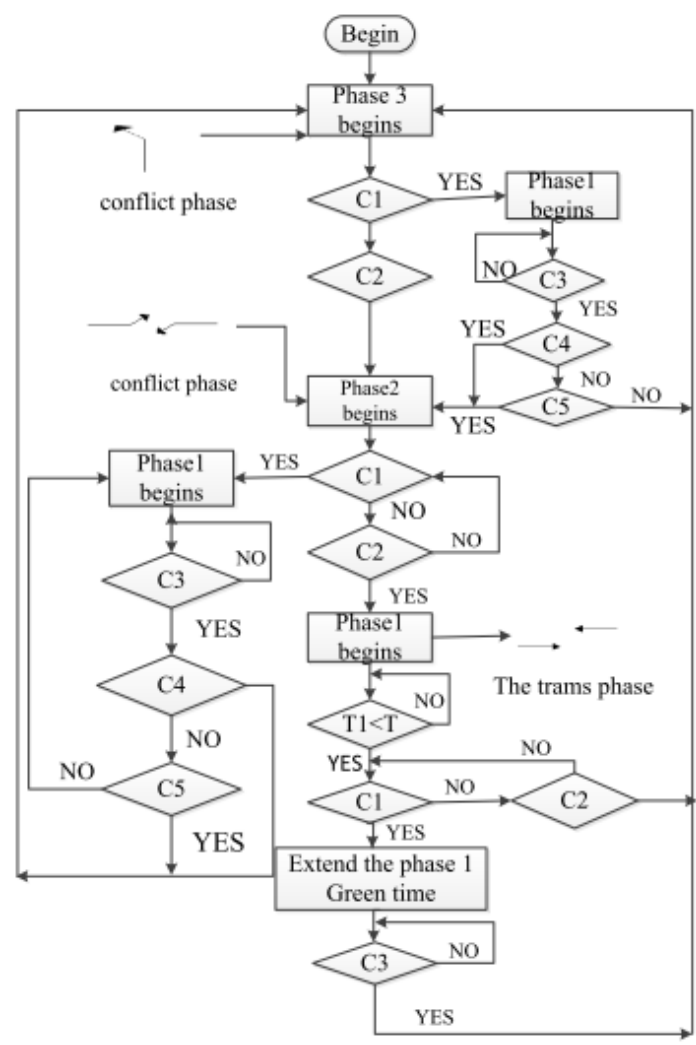

Fig.3 Schematic diagram of control scheme

Inductive signal control type. Trams signal priority control scheme relies on inductive signal control implementation. Inductive control is divided into three categories: optimization of induction, full induction, half induction control. Based on the principle design of inductive signal which including three kinds of green time: initial green time, unit green extension time, green light time limit extension. Various green time affected factors is not the same. When the initial phase is the green light, setting up an 'initial green time' in advance $\left(\mathrm{G}_{\min }\right)$. When the initial green time is in the end, there is no vehicle in the preset time interval that can replace phase right now. If once the subsequent vehicles are detected, according to the number of vehicles, it will extend the predetermined 'unit green extension time' automatically $\left(\mathrm{G}_{0}\right)$. If there is no vehicle in the scheduled time interval, the phase will be forced to interrupt. If there still have vehicles, then the green time will continue to extend. When the green time continues to the original set of 'green limit extension time' $\left(G_{\max }\right)$, even if a vehicle is detected, it will also return the way to finish the phase forcefully. The actual green time scope is between the initial green time and green limit extension time. This scheme applies the optimal inductive signal control type.

Priority control overall design figure. According to the design scheme of sensing detector and signal light group settings, as Fig. 3 is shown, among them, $K_{i}(i=1,2 \ldots n)$ represents the signal light group, they can be understood as group phases. $D_{j}(j=1,2 \ldots n)$ represents the first $j$ detector of tram phase detector.

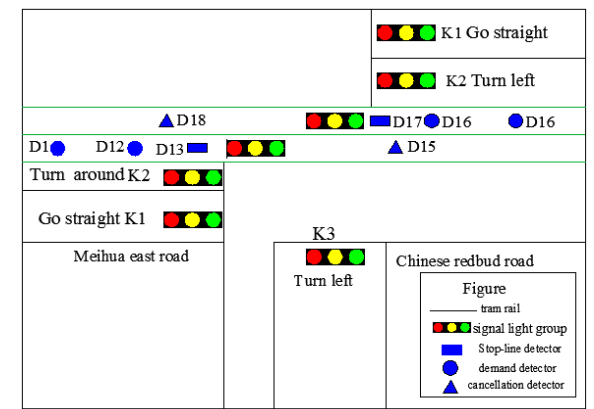

Fig.4 Schematic diagram of signal and detector layout 
VISSIM-VisVAP microscopic simulation analysis and evaluation. On the basis of the inductive signal control design, with the aid of VISSIM - VisVAP simulation platform, we compare Meihua east road - Chinese redbud road intersection's timing control with the active priority control. The evaluation parameters is divided into two classes, the first one is adapting travel time evaluation index for trams, the second one is using delay time and queue length two evaluation indexes for transverse normal social vehicles. The result is:

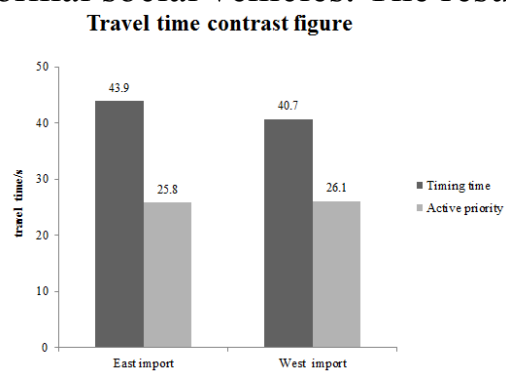

(a)Tram

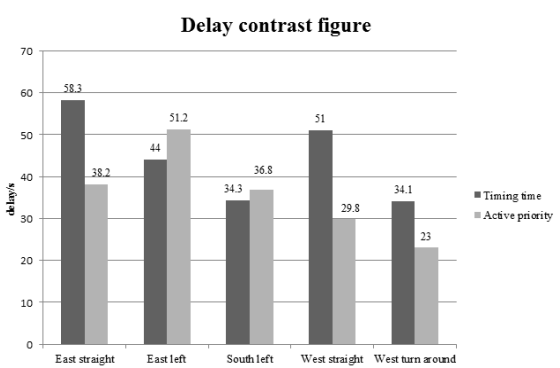

(b) Vehicle on the intersecting road

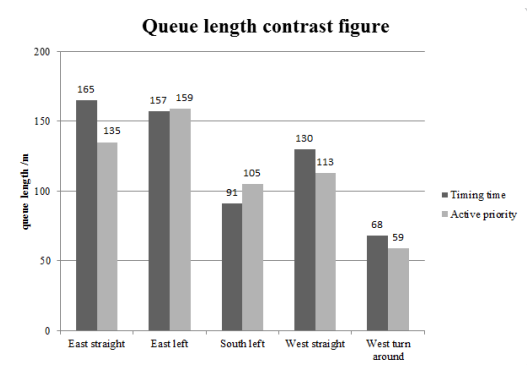

(c)Vehicle on the intersecting road

Fig.5 Comparison analysis of Fixed-time control and active priority control

\section{Conclusions}

Under the inductive control of active priority signal control efficiency for trams and the operation efficiency of motor vehicles which is synthetic released are all higher than timing control significantly. The ability of intersecting roads is influenced certainly. The control strategy and the implementation method with good generalization and can be used in other intersection along the road.

\section{Acknowledgements}

This work was financially supported by the Beijing Institute of Technology Zhuhai Natural Science Foundation (XK-2015-004).

\section{References}

[1]SUN, Ji-liang. Research on modern tram signal system and key technology. Railway Signaling \& Communication Engineering, 2013; 10(4):55-59;

[2] YU Hong-zhi, SUN Ji-liang, SHEN Da-chuan. Signal communication system and intelligent transportation system of the tram. Urban Transport of China, 2013; 11(4):44-51;

[3] MA, Wan-jing, YANG Xiao-guang. A Review of prioritizing signal strategies for bus services. Urban Transport of China, 2010; 8(6):70-78,16;

[4] TANG Miao, MA Yun. Adaptability of modern tram in cities. Journal of Shanghai Jiaotong University, 2011, (Z1):71-75;

[5] LI Kai, MAO Li-liang, ZHANG Hui, et al. Study on the control projects for modern tramcars at signal intersections. Urban Rapid Rail Transit, 2013; 26(2):104-107;

[6]Yann Wadjas, Peter G Furth. Transit signal priority along an arterial using advanced detection. TRB Annual Meeting, Washington DC, National Research Council, 2003;

[7]LIU Hong-chao, Alexander Skabardonis, ZHANGWei-bin. A dynamic model for adaptive bus signal priority. TRB Annual Meeting, Washington DC, National Research Council, 2003;

[8]Matthew Gatenby, Stephen Fedzin. Traffic signal network operation within the Nottingham express transit system. Traffic Engineering\&Control, 2004; 45(2):44—49. 